\title{
Light shift of the Ramsey resonance of coherent population trapping in optically dense medium
}

\author{
Konstantin Barantsev*, Gavriil Voloshin, Andrey Litvinov, and Evgeniy Popov \\ Peter the Great St. Petersburg Polytechnic University, Department of theoretical physics, 195251 \\ Saint-Petersburg, Polytechnicheskaia str., 29, Russia
}

\begin{abstract}
In this work we calculate the Ramsey resonances of coherent population trapping effect in cold atomic ensembles. The main goal is to investigate the light shift of the resonance on the different parameters in optically dense medium, because such medium can to distort and absorb the interrogating laser pulses.
\end{abstract}

Coherent population trapping (CPT) effect [1,2] allows us to detect the microwave transitions in atomic ensembles only by radiation of optical range. The two-frequency laser radiation interacts in simply case with the three-level $\Lambda$-system and induces the microwave atomic coherence. Atoms cease to scatter the light and the transparency window appears. Due to the fact that the microwave atomic coherence is long lived, the width of the transparency window is small compared with natural line width. It makes it possible to use this effect in wide range of practical applications such as quantum frequency standards [3], magnetometers [4], recording and processing of quantum information [5].

One of the ways to improve the accuracy of the metrological devices is to reduce the width of the reference resonance. Detection of the CPT resonance by the Ramsey method [6] allows us to further narrow the resonance line. The idea of this method consists of interrogation the atoms by the radiation not continuously, but in discrete periods. It can be done both by spatial separation of the interaction regions $[7,8]$ and by using pulsed radiation [9-12]. The Ramsey method using pulsed radiation has been actively researched in the last years. One of the main problems affecting the accuracy of the atomic clock is the light shift of the reference resonance. At present, a new direction of research is the search for ways to suppress the light shifts of CPT-resonance when using the Ramsey method by varying of the shapes of the pulses, their phases and amplitudes - the so-called generalized auto-balanced Ramsey spectroscopy [13]. The work [14] uses a two-stage pulse shape to simultaneously suppress light shifts and improve the signal-to-noise ratio. In [15] the density matrix formalism is constructed for atoms in an arbitrary periodic field.

Despite the large number of modern works on the investigation of Ramsey detection method, there remain some open questions related to the influence of an optical density [16] of the medium at the Ramsey signal. The optically dense medium is the atomic medium where the mean free path of the quasiresonant photon is much smaller than the size of the medium. In such medium the laser radiation can be absorbed significantly, the form of the

\footnotetext{
* Corresponding author: kostmann@yandex.ru
} 
interrogation laser pulses can be distorted and different modes are absorbed in different ways. This can drastically affect the total light shift of the CPT resonance, which is not taken into account in modern theoretical works in this direction. Moreover, the effects of dense medium can suggest new ways of suppressing the light shift of the Ramsey signal. The radiation modes can be equalized in intensity due to a different absorption coefficient, which will result in an auto-balancing effect. Such effect has already been observed by us in the excitation of the CPT resonance by continuous radiation [17].

In this work we investigate the influence of the optically dense medium on the light shift of the Ramsey resonance of coherent population trapping in cold atomic ensembles. The dependencies of the light shift on the intensity of radiation, durations of the laser pulses and dark pause, dipole moments of the atomic transitions, length of the medium and atomic concentration have been found.

This work is supported by Ministry of Education and Science of the Russian Federation in terms of FTP "Research and development on priority trends of Russian scientific-technological complex evolvement in 2014-2020 years (agreement 14.578.21.0211, agreement unique identifier RFMEFI57816X0211)".

\section{References}

1. G. Alzetta, A. Gozzini, L. Moi et al., Nuovo Cimento B, 36, 5 (1976)

2. E. Arimondo, G. Orriols, Nuovo Cimento Lett., 17, 333 (1976)

3. J. Vanier, Appl.Phys. B, 81, 421 (2005)

4. A. Akulshin, A. Celikov and V. Velichansky, Opt.Commun., 84, 139 (1991)

5. M. Fleishhauer and M. D. Lukin, Phys. Rev. Lett., 84, 5094 (2000)

6. N. F. Ramsey, Phys. Rev., 78, 695 (1950)

7. J. E. Thomas, P. R. Hemmer, S. Ezekiel et al., Phys. Rev. Lett., 48, 867 (1982)

8. I. M. Sokolov, Quantum Electronics, 45, 947 (2015)

9. G. Pati, K. Salit, R. Tripathi et al., Opt. Commun., 281, 4676 (2008)

10. Y. Yano, W. Gao, S. Goka, and M. Kajita, Phys. Rev. A, 90, 013826 (2014)

11. G. S. Pati, Z. Warren, N. Yu, and M. S. Shahriar, JOSA B, 32, 388 (2015)

12. P. Yun, F. Tricot, C. E. Calosso et al., Phys. Rev. Applied, 7, 014018 (2017)

13. T. Zanon-Willette, V. I. Yudin and A. V. Taichenachev, Phys. Rev. A, 92, 023416 (2015)

14. Y. Yano, S. Goka, M. Kajita, arXiv: 1701.05696v1 (2017)

15. V. I. Yudin, A. V. Taichenachev, M. Yu. Basalaev, Phys. Rev. A, 93, 013820 (2016)

16. M. B. Gornyi, B. G. Matisov, Yu. V. Rozhdestvenskii, Zh. Eksp. Teor. Fiz., 95, 1263 (1989)

17. K. A. Barantsev, A. N. Litvinov, E. N. Popov, I. M. Sokolov, IEEE Xplore, DOI: 10.1109/EFTF.2016.7477818 (2016) 